\title{
Correction to: Comparative transcriptomics reveals PrrABmediated control of metabolic, respiration, energy-generating, and dormancy pathways in Mycobacterium smegmatis
}

Jason D. Maarsingh ${ }^{1,2}$, Shanshan Yang ${ }^{3}$, Jin G. Park ${ }^{4}$ and Shelley E. Haydel ${ }^{1,5^{*}}$

\section{Correction to: BMC Genomics \\ https://doi.org/10.1186/s12864-019-6105-3}

Following the publication of the original article [1], the authors reported an error in Fig. 1 of the PDF version of their article. Due to a typesetting mistake, a previous version of the figure was placed in the PDF, which therefore did not match the correct Fig. 2 given in the HTML version.

The original article has been corrected.

\begin{abstract}
Author details
${ }^{1}$ School of Life Sciences, Arizona State University, Tempe, AZ, USA. ${ }^{2}$ Department of Obstetrics and Gynecology, College of Medicine-Phoenix, University of Arizona, Phoenix, AZ, USA. ${ }^{3}$ Bioinformatics Core, Knowledge Enterprise Development, Arizona State University, Tempe, AZ, USA. ${ }^{4}$ The Biodesign Institute Virginia G. Piper Center for Personalized Diagnostics, Arizona State University, Tempe, AZ, USA. ${ }^{5}$ The Biodesign Institute Center for Immunotherapy, Vaccines and Virotherapy, Arizona State University, Tempe, $A Z$, USA
\end{abstract}

Published online: 31 December 2019

\section{Reference}

1. Maarsingh, et al. Comparative transcriptomics reveals PrrABmediated control

of metabolic, respiration, energy-generating, and dormancy pathways in Mycobacterium smegmatis. BMC Genomics. 2019;20:942 https://doi.org/10. 1186/s12864-019-6105-3.

* Correspondence: Shelley.Haydel@asu.edu

${ }^{1}$ School of Life Sciences, Arizona State University, Tempe, AZ, USA

${ }^{5}$ The Biodesign Institute Center for Immunotherapy, Vaccines and

Virotherapy, Arizona State University, Tempe, AZ, USA

Full list of author information is available at the end of the article

(c) The Author(s). 2019 Open Access This article is distributed under the terms of the Creative Commons Attribution 4.0 International License (http://creativecommons.org/licenses/by/4.0/), which permits unrestricted use, distribution, and reproduction in any medium, provided you give appropriate credit to the original author(s) and the source, provide a link to the Creative Commons license, and indicate if changes were made. The Creative Commons Public Domain Dedication waiver (http://creativecommons.org/publicdomain/zero/1.0/) applies to the data made available in this article, unless otherwise stated. 
The incorrect figure was:

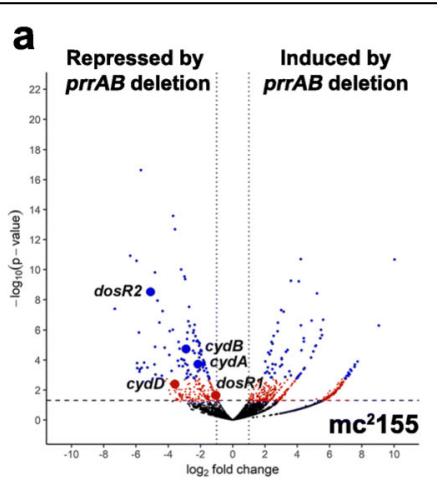

b
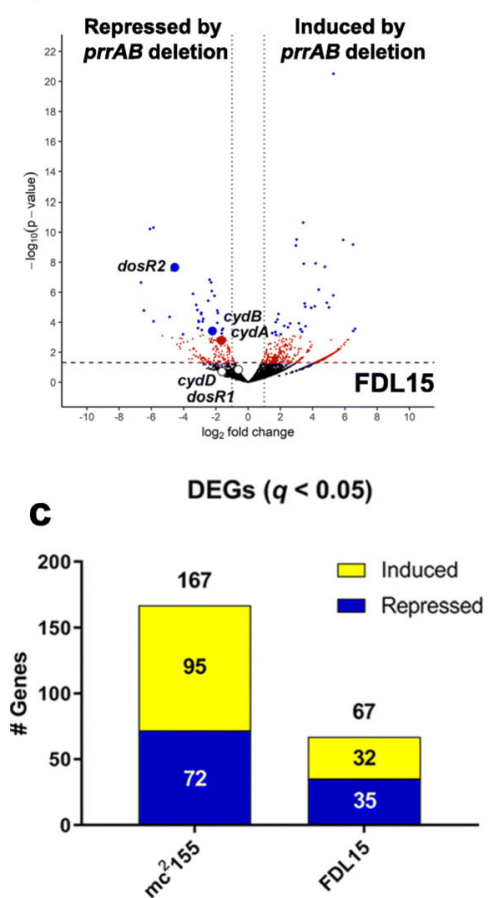

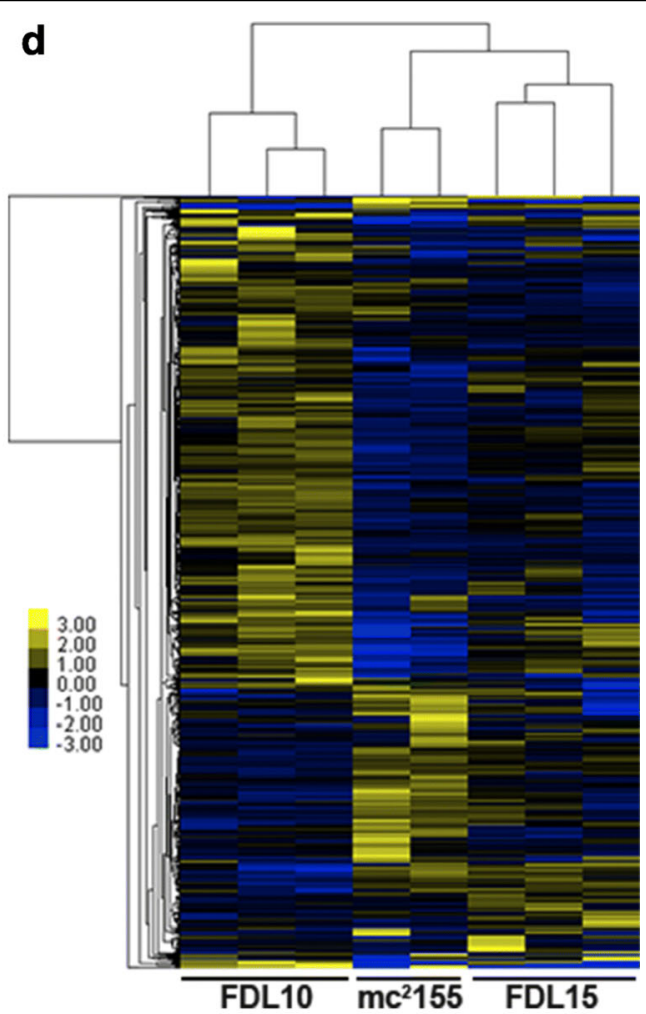

e

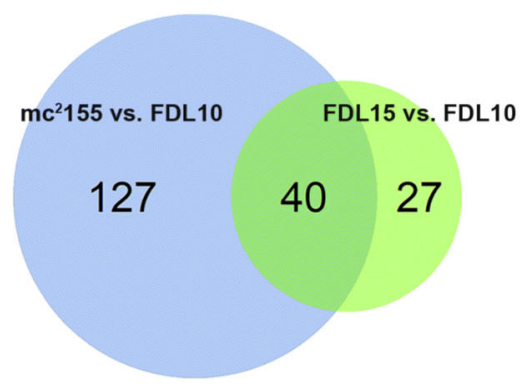

Fig. 2 Global DEG profiles $(q<0.05)$ between the mc2 155 vs. FDL10 and FDL15 vs. FDL10 RNA-seq comparisons. Volcano plots of (a) FDL10 vs. mc2 155 and (b) FDL10 vs. FDL15 group comparisons with red and blue dots representing differentially-expressed genes with $p<0.05$ and $q<$ 0.05 , respectively. The horizontal hatched line indicates $p=0.05$ threshold, while the left and right vertical dotted lines indicate log 2 fold change of -1 and +1 , respectively. c Repressed (blue) and induced (yellow) DEGs ( $q<0.05$ ) in mc2 155 (WT) and FDL15 (prrAB complementation strain) compared to the FDL10 $\triangle$ prrAB mutant. d Average hierarchical clustering (FPKM + 1) of individual RNA-seq sample replicates. e Venn diagrams indicating 40 overlapping DEGs (q < 0.05) between mc2 155 vs. FDL10 (WT vs. $\Delta$ prrAB mutant) and FDL15 vs. FDL10 (prrAB complementation strain vs. $\triangle$ prrAB mutant) strain comparisons 
The correct figure is:
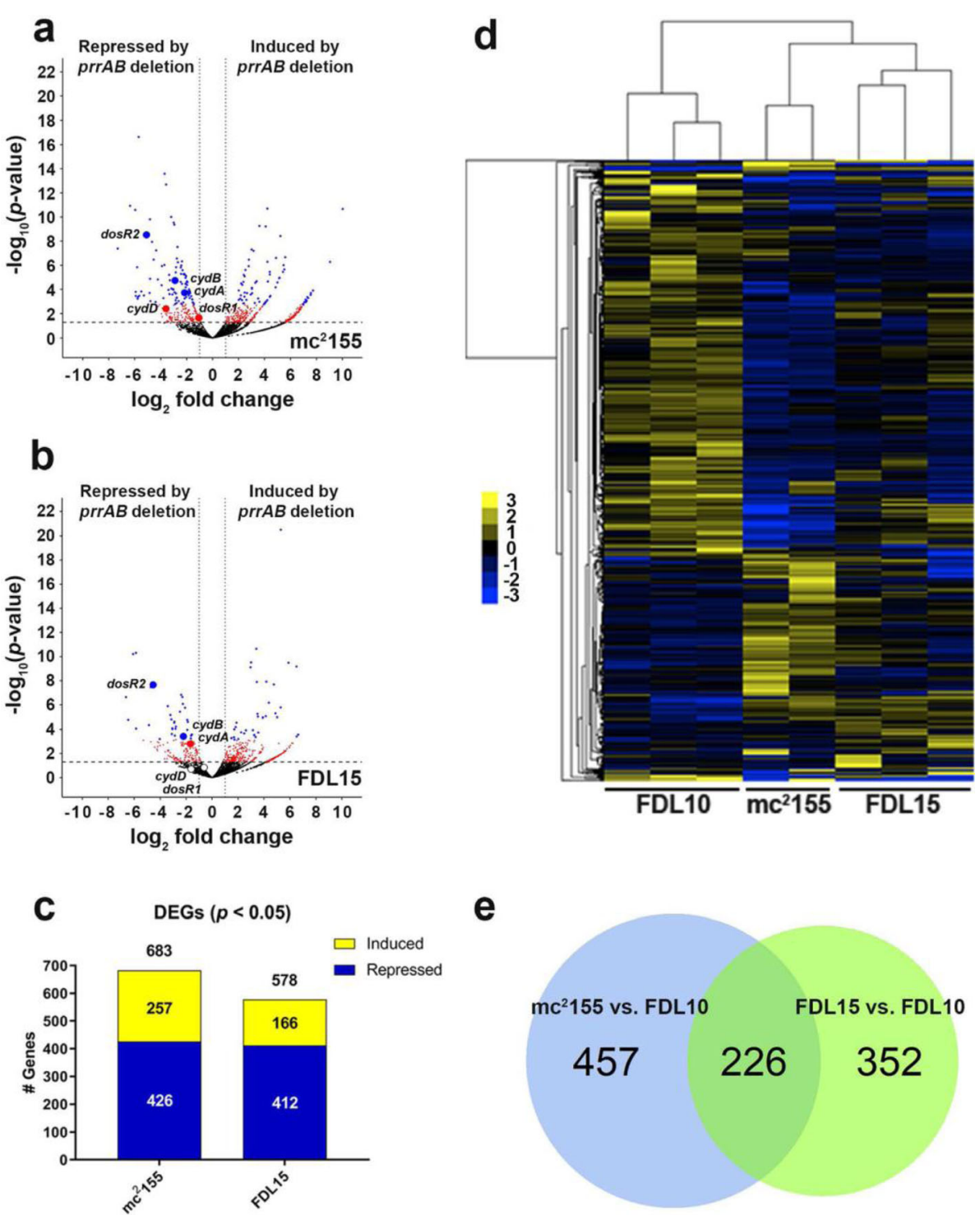

Fig. 2 Global DEG profiles $(q<0.05)$ between the mc2 155 vs. FDL10 and FDL15 vs. FDL10 RNA-seq comparisons. Volcano plots of (a) FDL10 vs. mc2 155 and (b) FDL10 vs. FDL15 group comparisons with red and blue dots representing differentially-expressed genes with $p<0.05$ and $q<0.05$, respectively. The horizontal hatched line indicates $p=0.05$ threshold, while the left and right vertical dotted lines indicate log2 fold change of -1 and +1 , respectively. c Repressed (blue) and induced (yellow) DEGs $(q<0.05)$ in mc2 155 (WT) and FDL15 (prrAB complementation strain) compared to the FDL10 $\triangle$ prrAB mutant. d Average hierarchical clustering (FPKM + 1) of individual RNA-seq sample replicates. e Venn diagrams indicating 40 overlapping DEGs (q < 0.05) between mc2 155 vs. FDL10 (WT vs. $\triangle$ prrAB mutant) and FDL15 vs. FDL10 (prrAB complementation strain vs. $\triangle$ prrAB mutant) strain comparisons 